of pedigree herds make sure that any pigs about the place are free from tuberculosis. Sheep for some unknown reason are remarkably free from it. Apart from harbouring parasitic worms, the cats and dogs on a farm are usually healthy, though like most other mammals these animals anywhere can be infected with tubercle if in contact with it.

Unless there is some special reason, now that cattle as a whole are free from tuberculosis the usual practice is to test annually, or at two-year intervals if the local health position is good. Happily the number of reactors remains very small out of a total cattle population of nine millions. When any reactors are discovered, all ordinary causes are fully investigated by the Ministry of Agriculture veterinary staff. A common trouble stems from animals with longstanding, but not necessarily severe, disease in which foci of potentially pathogenic bacilli have become completely encysted. Lesions may remain in this state for the whole of an animal's life and may be noticed only when it is eventually slaughtered. Some beasts in this state completely lose their cutaneous tuberculin test reactions. Owing to some unfavourable condition of life, or even calving stress, occult foci of infection can break down and active disease become re-established, with serious results. Difficulties sometimes arise about the correct interpretation of reaction to infections with acid-fast bacilli not generally regarded as pathogenic. ${ }^{2}$ But better the slaughter of an occasional animal with a false-positive test than that the public health should suffer from a missed reactor.

If no ordinary cause can be found for the emergence of reactors on a farm, unusual possibilities have to be considered. This is when wild mammals become suspect. Recently in a few counties some pockets of infection have appeared which have defied explanation, and the most likely cause would seem to be a local spontaneous outbreak of disease in wild life. Even badgers have thus come under suspicion in one county. These animals are known to be particularly healthy, probably because of their standards of personal and domestic hygiene, and this is the first occasion when active tuberculosis has been demonstrated in some carcasses and droppings. The matter is being fully investigated by the Ministry of Agriculture. The question will have to be answered whether both cattle and badgers have become infected from a common source, such as rats or possibly a contaminated stream. Chance observations of this sort, of which the significance is not clear, provide no justification for the wholesale slaughter of these or other wild animals.

1 British Medical fournal, 1971, 2, 419.

2 Thrower, W. R., Fournal of the Royal College of Physicians, 1970, 4, 277.

\section{Inappropriate Secretion of ADH}

The syndrome of inappropriate secretion of antidiuretic hormone was first described in a patient with bronchial carcinoma. ${ }^{1}$ It has now been found in many other conditions, which apparently have few clinical features in common. Other diseases of the lung such as tuberculosis and pneumonia may give rise to it. ${ }^{2} \mathrm{~A}$ recent report from the Mayo $\mathrm{Clinic}^{3}$ describes three patients, one with staphylococcal pneumonia and two with viral pneumonia, who showed the typical features. Disorders of the nervous system, such as head injuries, cere- bral tumours, and brain abscesses, gastrointestinal lesions such as tumours of the small intestine and pancreas, all may cause a clinical picture suggestive of secretion of antidiuretic hormone. The syndrome has also been described in patients with myxoedema and acute porphyria and after cardiac bypass. ${ }^{4}$

The pathogenesis of inappropriate secretion of antidiuretic hormone in all these conditions is obscure. Some tumours produce antidiuretic hormone themselves, and the same has been shown to be true of tuberculous lung tissue 5 in one patient. Under physiological conditions the stimulus to secretion of the hormone is thought to be, firstly, changes in the volume of intravascular fluid 6 and, secondly, changes in osmolality of extracellular fluid. ${ }^{7}$ In animals it has been shown that inflation of a balloon within the left atrium results in an increase in rate of urine flow, whereas factors promoting pooling of blood within the periphery, and thus decreasing left atrial pressure, promote increased antidiuretic hormone activity and a decrease in the volume of urine. ${ }^{8}$ In man this association between left atrial pressure and secretion of the hormones is inferential. It has been suggested that in patients with pneumonia a decrease in cardiac output and acute hypoxia may lead to pulmonary vasoconstriction and thus decreased filling of the left atrium. ${ }^{3}$ It is also possible, though perhaps less likely, that infected lung tissue produces a substance resembling the antidiuretic hormone.

Whatever the cause, an oversecretion of the hormone leads to the retention of water, with resulting hyponatraemia. To protect the body against increases in fluid volume, increasing amounts of sodium are lost in urine, but this leads only to further decreases in the osmolality of the body fluids. Thus the increased urinary excretion of sodium is not due to any intrinsic defect in renal tubular function, since the sodium-retaining response to mineralocorticoids in these patients is intact. 9

Certain biochemical abnormalities give the clues to this syndrome, and they were present in the three patients described from the Mayo Clinic. They are, firstly, a fall in the concentration of serum sodium, resulting in a low serum osmolality; and, secondly, an increased urinary excretion of sodium resulting in urine which is hypertonic to plasma. The concenrtation of antidiuretic hormone in the plasma is difficult to assay, but it was raised in one of the Mayo clinic patients.

The clinical features of the syndrome can be considered under two headings. Firstly, there are the signs of the underlying disease; secondly, there are the symptoms of hyponatraemia. When the serum sodium falls to approximately $125 \mathrm{mEq} / \mathrm{L}$., the patient may have some non-specific symptoms-namely, anorexia, nausea, and vomiting. When the concentration falls below $115 \mathrm{mEq} / \mathrm{L}$. the patient becomes progressively excited, depressed, lethargic, and finally comatose. Generalized convulsions may be seen as a terminal event. Those patients with inappropriate secretion of antidiuretic hormone differ from patients with "true" sodium depletion in that such signs of fluid depletion as hypotension, uraemia, and poor skin tone are absent. They can also be distinguished from patients with hyponatraemia due to congestive heart failure or cirrhosis of the liver, who are oedematous, while the patients described here are not. The greatest differential diagnostic problem is the patient with adrenal insufficiency whose ability to excrete water is more impaired than his disorder of sodium metabolism. Thus hyponatraemia without hypotension occurs; only the appropriate tests of endocrine function will differentiate these two conditions. 
Treatment of patients with the syndrome is by fluid deprivation. This will usually cause a return of serum electrolytes to normal levels. The prognosis obviously depends on the nature of the underlying disease. It is of interest that the serum sodium concentration of patients with inappropriate secretion of antidiuretic hormone due to bronchial carcinoma may show a return to normal when the tumour is treated by radiotherapy. A high index of suspicion is necessary to make the diagnosis. As it becomes easier to measure plasma concentrations of antidiuretic hormone, more cases of inappropriate secretion of the hormone will probably be found.

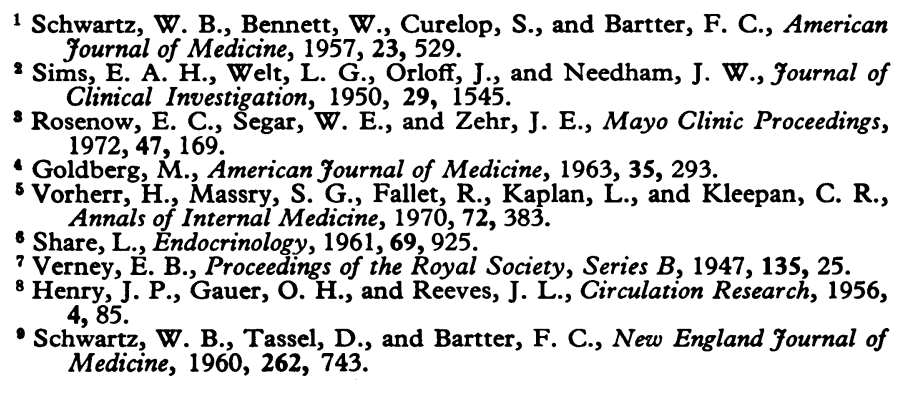

\section{Barbiturates in Asthma}

Phenobarbitone and other barbiturates are potent inducers of microsomal enzymes. These enzymes in the liver hydrolyse corticosteroids and thereby cut short the corticosteroids' biological activity.

The possibility that enzyme induction by phenobarbitone might adversely effect asthmatics receiving corticosteroids has been investigated recently by S. M. Brooks and his colleagues. ${ }^{1}$ They studied the effect of the drug, $120 \mathrm{mg}$ daily, in 11 asthmatic patients on isotope-labelled dexamethasone. The phenobarbitone decreased the half-life of the dexamethasone in the patients' plasma by $44 \%$ and increased the metabolic clearance by $88 \%$. These effects were not noted in five placebo-treated patients. Prednisone metabolism could not be studied directly because of the absence of a suitably labelled preparation. However, adverse effect of phenobarbitone on prednisone therapy could be inferred from observations on three prednisone-dependent patients. They showed increased asthmatic symptoms, deterioration of response in spirometric tests, and increased eosinophil counts when given phenobarbitone and improvement after the drug was withdrawn when reduction of prednisone dosage was possible. Patients not dependent on steroids did not show similar changes during phenobarbitone treatment.

Continual administration of phenobarbitone is therefore likely to reduce the therapeutic effect of corticosteroids in patients with asthma, and the prescribing of barbiturates might account for the need to increase the dosage of corticosteroids and cause the low plasma cortisol response which has been observed in some asthmatic patients. ${ }^{2}$ Many proprietary bronchodilator mixtures contain barbiturates. Moreover, intermittent use of barbiturates could result in instability of corticosteroid requirements in corticosteroiddependent patients and difficulty in tapering off dosage. Other hypnotics known to be enzyme-inducers in man include dichloralphenazone ${ }^{3}$ and glutethimide, both of which might have a similar adverse effect in corticosteroid-dependent patients. Severely ill asthmatic patients should not be given sedatives, since in respiratory failure there is probably no drug with sedative, hypnotic, or tranquillizing properties which cannot cause respiratory depression. 45

Drugs known to cause enzyme induction in animals include sedatives, hyponotics, some anaesthetic gases, tranquillizers, antidepressants, analgesic, anti-inflammatory and uricosuric drugs, sulphonylureas, analeptics, anticonvulsants, muscle relaxants, antihistamines, and corticosteroids. There is a lamentable lack of knowledge of the extent and significance of enzyme induction in man and its therapeutic implications. Yet these may be as important as, for instance, the interaction between oral anticoagulants and barbiturates. Such pharmacokinetic drug interactions are likely to make the simultaneous prescription of several drugs increasingly hazardous. ${ }^{6}$ 1 Brooks, S. M., Werk, E. E., Ackerman, S. J., Sullivan, I., and Dwyer, J., Lazarus, L., and Hickie, J. B., Australasian Annals of Medicine, 1967, 16, 297.

3 Brecken-idge, A., Orme, M. L'E., Thorgeirsson, S., Davies, D. S., and Brooks, R. V., Clinical Science, 1971, 40, 351.

4 Clark, T. J. H. Collins, J. V., and Tong, D., Lancet, 1971, 2, 737.

5 Gaddie, J., Legge, J. S., Palmer, K. N. V., Petrie, J. C., and Wood, R. A., British Medical fournal, 1972, 2, 688.

6 Prescott, L. F., Lancet, 1969, 2, 1239.

\section{Study of Ethics}

When medicine was learnt essentially by apprenticeship, students picked up some ethical guidance along with the techniques of taking a history or palpating the abdomen. They might end up by reciting, or acknowledging in solemn silence, a version of the Hippocratic Oath-or more probably they might not. And when the time came for independent practice the ethical rules underlying it seemed natural, practical, and clear. But the growing power of medicine has meant that doctors can now influence-have an obligation to influence-much that was formerly left to nature or providence. The founding of the Society for the Study of Medical Ethics is therefore timely.

The aim of the new society is to promote the study of medical ethics. It hopes to do this by sponsoring an annual conference, organizing occasional meetings, and by encouraging study of the subject in other ways. Further details may be obtained from the secretary of the society at 103 Gower Street, London W.C.1.

If this society can avoid becoming a talking-shop for the airing of opinions on matters about which none can be an expert, it should help to concentrate doctors' attention on the many contentious issues that now affect their patients' welfare. The beginning of life and its ending are both presenting new ethical problems, while the quality of life in between is also being considered. Lunchtime abontions, organ transplants, resuscitation, even the prescribing of an antibiotic to a defective baby or to a sinking old person are among the variety of circumstances that have stirred arguments on ethics in recent years. Difficult decisions can worry every one in clinical practice with remarkable suddenness. 